\title{
Backpropagation of Physiological Spike Trains in Neocortical Pyramidal Neurons: Implications for Temporal Coding in Dendrites
}

\author{
Stephen R. Williams and Greg J. Stuart \\ Division of Neuroscience, John Curtin School of Medical Research, Australian National University, Canberra, Australian \\ Capitol Territory 0200, Australia
}

\begin{abstract}
In vivo neocortical neurons fire apparently random trains of action potentials in response to sensory stimuli. Does this randomness represent a signal or noise around a mean firing rate? Here we use the timing of action potential trains recorded in vivo to explore the dendritic consequences of physiological patterns of action potential firing in neocortical pyramidal neurons in vitro. We find that action potentials evoked by physiological patterns of firing backpropagate threefold to fourfold more effectively into the distal apical dendrites ( $>600 \mu \mathrm{m}$ from the soma) than action potential trains reflecting their mean firing rate. This amplification of backpropagation was maximal during high-frequency components of physiological spike trains $(80-300 \mathrm{~Hz})$. The disparity between backpropagation during physiological and mean firing patterns was dramatically reduced by dendritic hyperpolarization. Consistent with this voltage dependence, dendritic depolarization amplified single action potentials by fourfold to seven-
\end{abstract}

fold, with a spatial profile strikingly similar to the amplification of physiological spike trains. Local blockade of distal dendritic sodium channels substantially reduced amplification of physiological spike trains, but did not significantly alter action potential trains reflecting their mean firing rate. Dendritic electrogenesis during physiological spike trains was also reduced by the blockade of calcium channels. We conclude that amplification of backpropagating action potentials during physiological spike trains is mediated by frequency-dependent supralinear temporal summation, generated by the recruitment of distal dendritic sodium and calcium channels. Together these data indicate that the temporal nature of physiological patterns of action potential firing contains a signal that is transmitted effectively throughout the dendritic tree.

Key words: sodium channel; patch clamp; action potential; neocortex; dendrite; firing pattern
Neurons generate a barrage of action potentials with variable instantaneous frequencies in response to sensory stimuli. These observations have raised an important question in neurobiology: what property of the output spike train encodes afferent information? Two opposing answers to this question have been suggested: (1) that the timing of action potentials precisely encodes afferent input on a spike by spike basis, and (2) that variations in action potential timing is merely a reflection of noise around a mean firing rate (Softky and Koch, 1993; Mainen and Sejnowski, 1995; Shadlen and Newsome, 1995; Softky, 1995; Shadlen and Newsome, 1998; Stevens and Zador, 1998).

Action potentials in cortical pyramidal neurons are initiated in the axon and propagate both axonally and back into the dendritic tree (Stuart and Sakmann, 1994; Spruston et al., 1995; Buzsaki and Kandel, 1998). The active dendritic backpropagation of action potentials is thought to provide an important retrograde message, signaling the activity state of the neuron (Johnston et al., 1996; Stuart et al., 1997b). Previous investigations, however, have indicated that the amplitude of backpropagating action potentials in the distal dendrites of cortical pyramidal neurons dramatically decreases during fixed frequency trains (Callaway and Ross, 1995; Spruston et al., 1995; Colbert et al., 1997; Stuart et al., 1997a), suggesting that action potential backpropagation during sustained action potential firing will be minimal at distal dendritic sites. Little is known, however, about the reliability of dendritic action potential backpropagation during physiological patterns of action potential firing, which are characterized by highly variable firing rates.

To directly address this question, we have reproduced physiological action potential firing patterns recorded in vivo in neocortical neurons maintained in slice preparations in vitro and investi-

Received June 8, 2000; revised Aug. 16, 2000; accepted Aug. 24, 2000.

This work was supported by a grant from the Wellcome Trust. We thank Dr. W. T. Newsome for sharing his data on spike timing.

Correspondence should be addressed to Dr. Greg J. Stuart, Division of Neuroscience, John Curtin School of Medical Research, Mills Road, Australian National University, Canberra, A.C.T. 0200, Australia. E-mail: Greg.Stuart@anu.edu.au.

Copyright (C) 2000 Society for Neuroscience $0270-6474 / 00 / 208238-09 \$ 15.00 / 0$ gated their impact on the dendritic tree. We demonstrate that physiological trains of action potentials effectively engage dendritic electrogenesis in the distal dendrites of layer 5 pyramidal neurons. As a consequence of their variable frequency content physiological, but not regular trains of action potentials, are capable of dramatic amplification produced by the recruitment of distal dendritic sodium and calcium channels. Furthermore, we investigate the dependence of dendritic backpropagation on stimulus frequency and membrane potential and directly measure dendritic sodium channel inactivation during action potential like trains. This work extends previous analysis of the frequency dependence of action potential backpropagation in cortical neurons and indicates that variations in instantaneous frequency during physiological patterns of action potential firing may have an important role in dendritic signaling.

\section{MATERIALS AND METHODS}

Wistar rats (3- to 5-weeks-old) were anesthetized by inhalation of Halothane, were decapitated, and 300- $\mu \mathrm{m}$-thick coronal neocortical brain slices were prepared, according to guidelines approved by the Animal Experimentation Ethics Committee of the Australian National University. Slices were perfused with an oxygenated solution of composition (in mM): 125 $\mathrm{NaCl} ; 25 \mathrm{NaHCO}_{3} ; 3 \mathrm{KCl} ; 1.25 \mathrm{NaH}_{2} \mathrm{PO}_{4} ; 2 \mathrm{CaCl} ; 1 \mathrm{MgCl}$ and 25 glucose. Somatic (pipette resistance 2-5 M $\Omega$ ) and dendritic (8-12 M $\Omega$ ) whole-cell current-clamp recordings were made from visually identified large layer 5 pyramidal neurons, as previously described (Stuart and Sakmann, 1994; Williams and Stuart, 1999). Somatic, dendritic, and axonal cell-attached recordings were made using pipettes of similar resistance (10-12 M $\Omega$ ) and on-line leak subtraction $(\mathrm{P} / 4)$. No differences in the degree or time of negative pressure applied to the back of pipettes was required to form high-resistance (3-10 G $\Omega$ ) seals at somatic, dendritic, or axonal sites, suggesting that similar membrane areas were sampled during cell-attached recordings (Williams and Stuart, 2000). All recordings were made at $35-36^{\circ} \mathrm{C}$. For whole-cell recordings patch electrodes were filled with (in mM): $135 \mathrm{~K}$-gluconate, $7 \mathrm{NaCl}, 10 \mathrm{HEPES}, 2 \mathrm{Na}_{2}$ ATP, $0.3 \mathrm{Na}_{2} \mathrm{GTP}$, and $2 \mathrm{MgCl}, \mathrm{pH} 7.2$, adjusted with KOH (osmolarity $280 \mathrm{mOsm}$ ). For cellattached recordings patch electrodes contained (in mM): $120 \mathrm{NaCl}, 30$ tetraethylammonium chloride, 5 4-aminopyridine, $2 \mathrm{CaCl}, 1 \mathrm{MgCl}, 1$ $\mathrm{CdCl}$, and 10 HEPES, pH 7.2, adjusted with $\mathrm{NaOH}$, to pharmacologically block potassium and calcium currents. Action potentials were evoked antidromically by electrical stimuli $(<0.5 \mathrm{~mA},<100 \mu \mathrm{s})$ delivered by a patch-pipette placed under visual guidance close to the axon $(\sim 5 \mu \mathrm{m})$, 

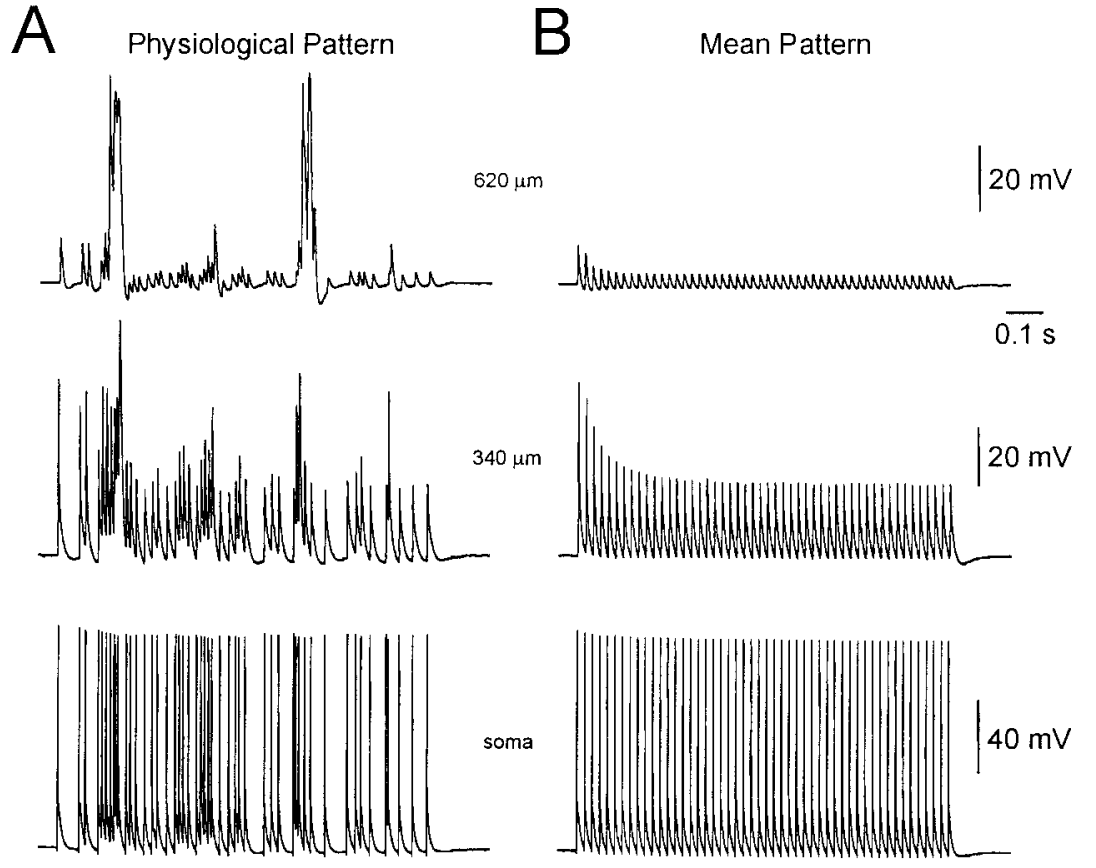

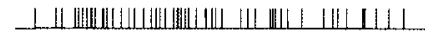

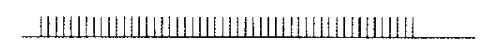

C

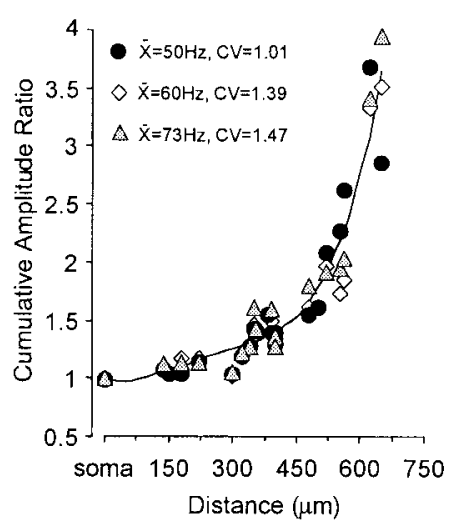

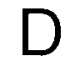

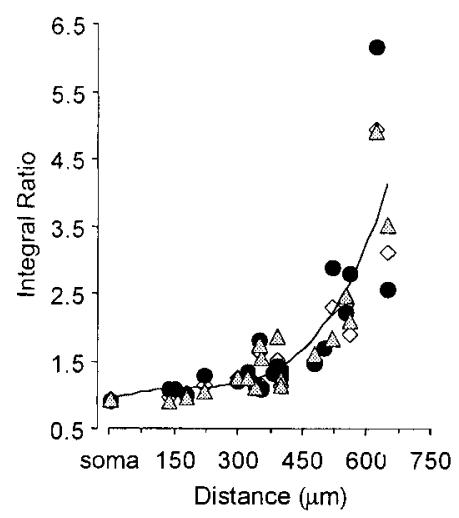

Figure 1. Physiological spike trains generate powerful dendritic electrogenesis. $A$, Antidromically evoked action potentials recorded at the soma and two dendritic locations (340 and $620 \mu \mathrm{m}$ from the soma) in response to a physiological spike train with mean frequency of $50 \mathrm{~Hz}$ and $\mathrm{CV}$ of 1.01 . B, Action potentials recorded at these locations when evoked regularly at the same mean frequency $(50 \mathrm{~Hz})$. All recordings in $A$ and $B$ were made from the resting membrane potential. Spike trains are shown at the bottom for clarity. $C$, Ratio of cumulative action potential amplitude of all action potentials in physiological relative to mean trains as a function of distance from the soma. Data from 25 neurons during three different physiological and mean trains (inset). The data were approximated with an arbitrary fourth order polynomial function constrained at the level of the soma. $D$, Ratio of the integral of all action potentials in physiological relative to mean trains as a function of distance from the soma. Data from 25 neurons during three different physiological and mean trains (see inset in $C$ ). Data were approximated with an arbitrary fourth order polynomial function constrained at the level of the soma.
20-30 $\mu \mathrm{m}$ from the soma. Antidromic stimulation was used to allow the accurate timing of action potential generation. Great care was taken to elicit pure antidromic responses. The absence of synaptic responses was verified by searching for their existence at stimulus intensities set just subthreshold for the generation of antidromic action potentials across a wide membrane potential range in each recording. Furthermore, similar results were observed in the presence of kynurenic acid $(3 \mathrm{~mm} ; n=4)$.

Physiological spike trains, evoked by optimal contrast stimuli from neocortical neurons in area MT in awake unanesthetized monkeys, were obtained from published data from Dr. W. T. Newsome (http://www. cns.nyu.edu/home/wyeth/data/newsome/newsome.html). Three different physiological spike trains containing 50 action potentials were used with mean frequencies of 50,60 , and $73 \mathrm{~Hz}$ and coefficients of variation $(\mathrm{CV})$ of $1.01,1.39$, and 1.47 , respectively. Long $(30 \mathrm{sec})$ time periods were left between trains of action potentials to control for any effects of slow sodium channel inactivation (Fleidervish et al., 1996). The integral of action potential trains was measured from the peak of the integrated voltage waveform, a point that corresponded to the last action potential in a train. Tetrodotoxin (TTX) $(1 \mu \mathrm{M})$ dissolved in extracellular solution was applied locally to dendrites by pressure application. The application pipette (similar to that used for dendritic whole-cell recording $)$ was placed close $(\sim 10$ $\mu \mathrm{m}$ ) to the dendrite $20-30 \mu \mathrm{m}$ proximal to the dendritic recording site under visual guidance and brief positive pressure $(150-200 \mathrm{mmHg})$ applied, resulting in a spatially localized ejection area $(\sim 100 \mu \mathrm{m}$ diameter $)$ (Stuart and Sakmann, 1995; Magee and Johnston, 1997; Williams and Stuart, 1999). Voltage and current signals were filtered at $10-30 \mathrm{kHz}$ for whole-cell recordings or $2-5 \mathrm{kHz}$ for cell-attached recordings, and were acquired at $20-100 \mathrm{kHz}$ using an ITC-16 interface (Instrutech Corpora- tion) controlled by an Apple Power personal computer. In all records illustrated the stimulus artifact has been blanked for clarity. Numerical values are given in the text as mean \pm SEM. Statistical analysis was performed with Student's $t$ test $(\alpha=0.05)$.

\section{RESULTS}

\section{Effective dendritic backpropagation of physiological spike trains}

Physiological action potential trains recorded in vivo in response to sensory stimuli in neocortical neurons from awake monkeys were reproduced in layer 5 pyramidal neurons by antidromic stimulation in vitro. We compared the action potential discharge recorded at somatic and apical dendritic recording sites evoked by these physiological spike trains with those evoked by the same number of action potentials presented at the mean frequency (mean trains) (Fig. $1 A, B$ ). At the level of the soma the ratio of the cumulative amplitude of all action potentials in physiological and mean trains was found to be close to one $(1.0 \pm 0.003 ; n=5$; Fig. $1 C)$. In sharp contrast, at progressively distal dendritic recording sites the cumulative amplitude of physiological spike trains was found to be greater than those of matched mean trains, revealing an enhancement of backpropagation by threefold to fourfold at the most distal 


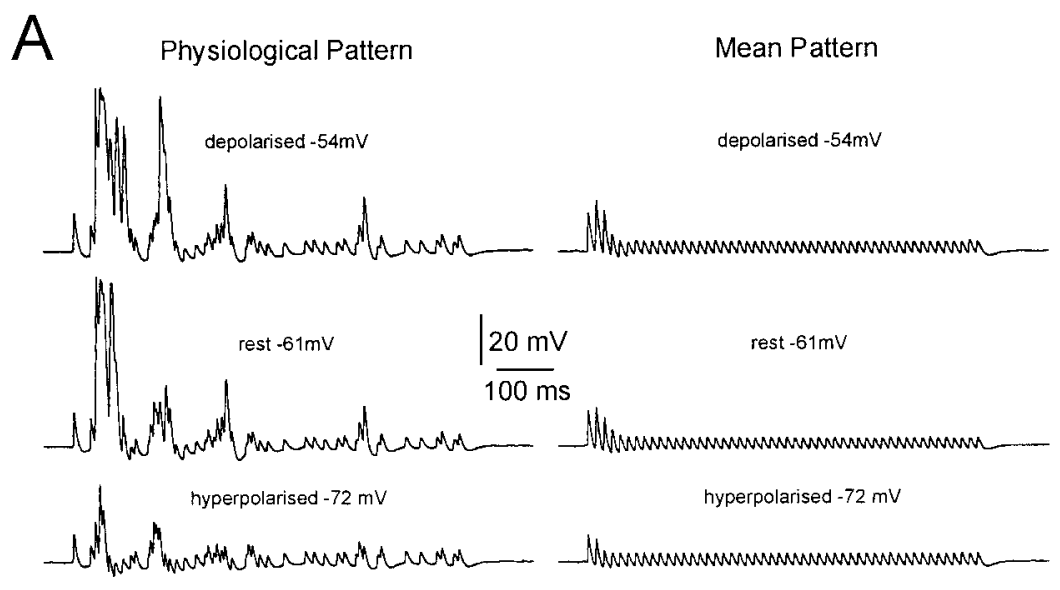

Figure 2. Voltage and frequency dependence of dendritic electrogenesis during physiological firing patterns. $A$, Voltage dependence of the disparity between physiological and mean trains. Dendritic action potentials generated during physiological trains (left traces) and mean trains (right traces) at depolarized (top traces), resting, and hyperpolarized (bottom traces) membrane potentials. The membrane potential was controlled by current injection through the dendritic recording electrode (560 $\mu \mathrm{m}$ from the soma). The physiological spike train shown had a mean frequency of $73 \mathrm{~Hz}$ and CV of 1.47. $B$, The integral ratio of physiological divided by the mean spike trains obtained at different membrane potentials is expressed as a fraction of the value at the resting membrane potential (represented as $0 \mathrm{mV}$ ). Note that membrane hyperpolarization greatly reduced this ratio. Data were approximated with an arbitrary second order polynomial function. $C$, Relationship between instantaneous firing frequency (gray symbols) and normalized dendritic action potential amplitude (black symbols) during the time course of a physiological spike train with a mean frequency of $50 \mathrm{~Hz}$ and $\mathrm{CV}$ of 1.01 . Action potential amplitude is expressed as a fraction of maximal amplitude and averaged for six dendritic recordings made $>480 \mu \mathrm{m}$ from the soma. $D$, Representative example demonstrating the relationship between dendritic action potential amplitude and instantaneous frequency, for the three different physiological firing patterns investigated. Dendritic recording made $620 \mu \mathrm{m}$ from the soma. $E$, Averaged data $(n=9$; all recordings $>450 \mu \mathrm{m}$ from the soma) of normalized distal dendritic action potential amplitude plotted as a function of the instantaneous action potential frequency for the three different physiological firing patterns investigated (amplitude normalized to the maximum amplitude in each trial for each neuron).
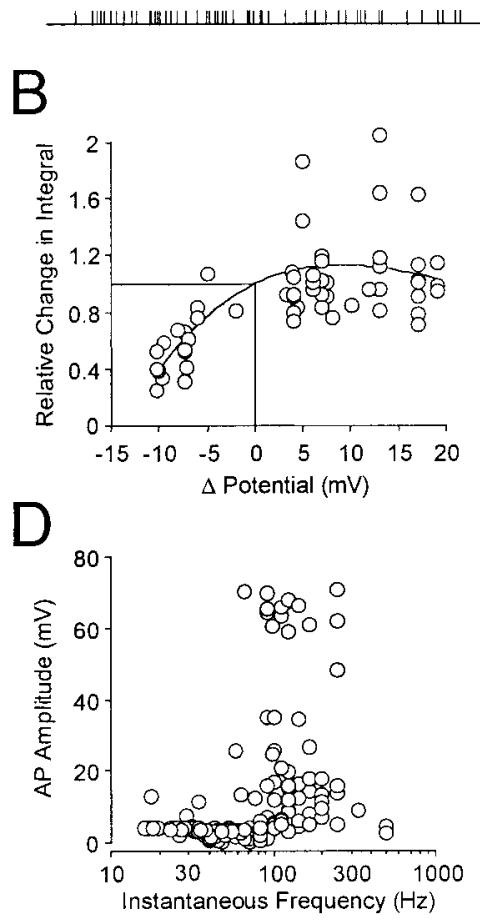

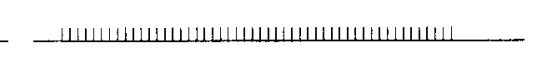

C

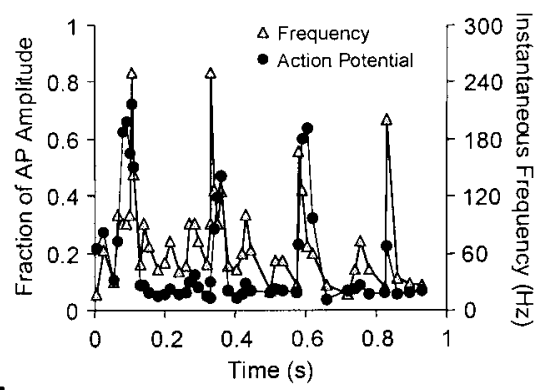

$\mathrm{E}$

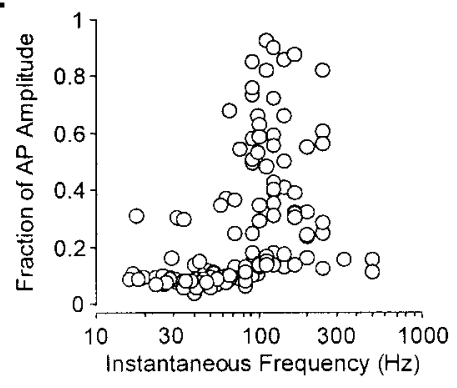

dendritic recording locations ( $>600 \mu \mathrm{m}$ from the soma; $n=20$; Fig. $1 C)$. Dendritic responses to physiological and mean action potential firing patterns were highly reproducible from trial to trial. A similar relationship was found when the integral of physiological and mean action potential trains were compared (Fig. 1D). These differences were attributable to periods of intense dendritic depolarization during physiological spike trains, where action potentials were observed to summate in a supralinear manner (Fig. 1A, top trace).

The membrane potential of layer 5 pyramidal neurons in vivo is depolarized relative to that in vitro, partly as a consequence of ongoing synaptic activity (Pare et al., 1998). We therefore explored the voltage dependence of the disparity between physiological and mean spike trains at distal dendritic recording sites by controlling the dendritic membrane potential by tonic current injection through the recording electrode $(n=10$; average recording distance from soma $486 \mu \mathrm{m}$; Fig. $2 A$ ). Theses experiments revealed that the relative difference between physiological and mean spike trains was maintained at membrane potentials up to $15 \mathrm{mV}$ depolarized to rest (Fig. $2 B$ ). In contrast, dendritic membrane hyperpolarization led to a dramatic reduction in the cumulative amplitude and integral of physiological spike trains (Fig. 2A,B). These data demonstrate that the selective amplification of physiological spike trains is apparent at physiologically relevant membrane potentials and that dendritic membrane hyperpolarization efficiently reduces this phenomena, indicating that the selective augmentation of physiological compared with mean action potential trains is generated by a dendritic voltage-dependent mechanism.

\section{Supralinear temporal summation of dendritic action potentials}

To explore the temporal nature of the enhancement of backpropagation during physiological spike trains, we analyzed the relationship between the amplitude of dendritic action potentials and the frequency of action potential generation (Fig. 2C). Throughout the time course of a physiological spike train dendritic action potential amplitude was found to map to changes in instantaneous frequency (calculated as the reciprocal of the interval between sequential action potentials). Dendritic action potential amplitude was found to be dramatically increased during time periods when the instantaneous action potential frequency was high (pooled data from six recordings made $>480 \mu \mathrm{m}$ from the soma; Fig. $2 C$ ). To explore this in more detail we plotted the amplitude of dendritic action potentials against instantaneous frequency (Fig. 2D,E). The amplitude of dendritic action potentials was found to be maximal when driven at instantaneous frequencies within a range of $80-300 \mathrm{~Hz}$ for each of the three different physiological trials (Fig. 2D, single neuron) and was consistent in all recordings made from distal dendritic locations $(n=9$; recordings $>450 \mu \mathrm{m}$ from the soma; Fig. $2 E)$. A strict 
A
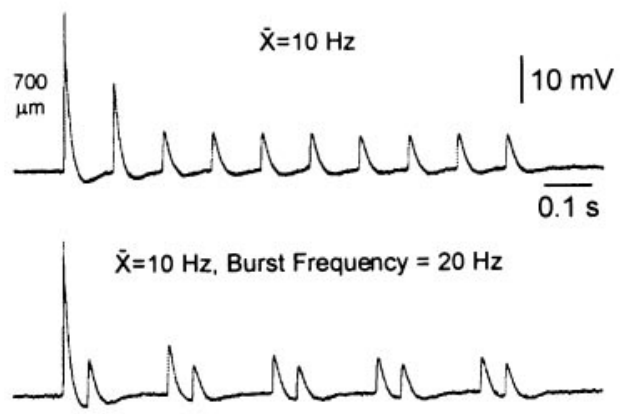

$\bar{X}=10 \mathrm{~Hz}$, Burst Frequency $=40 \mathrm{~Hz}$

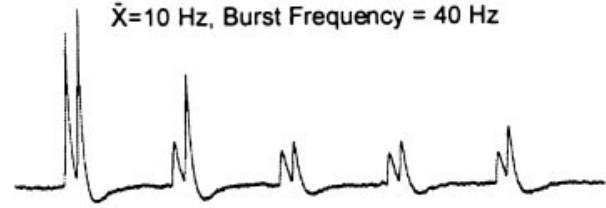

$\bar{X}=10 \mathrm{~Hz}$, Burst Frequency $=80 \mathrm{~Hz}$

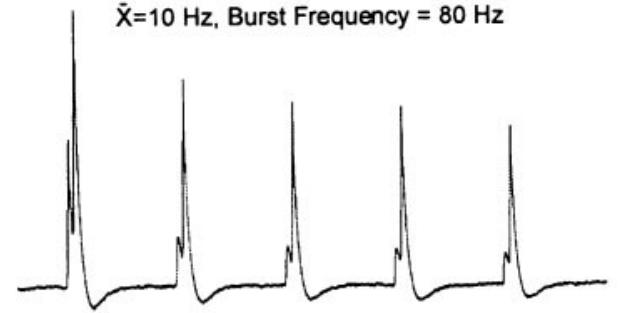

B
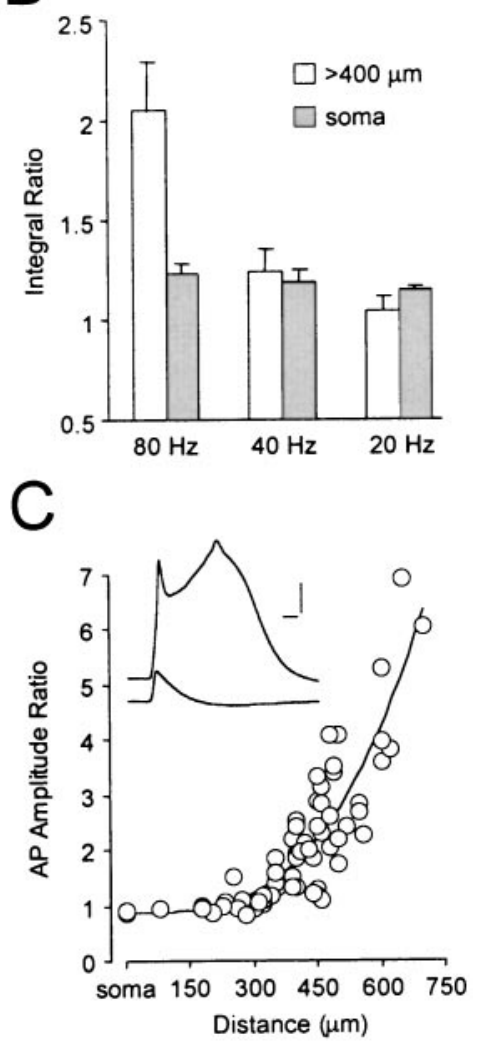

Figure 3. Supralinear temporal summation of dendritic action potentials. $A$, The top trace demonstrates activity-dependent depression of action potential amplitude during a regular frequency train $(10 \mathrm{~Hz}$; recording $700 \mu \mathrm{m}$ from the soma). Bottom traces show supralinear action potential temporal summation during trains with the same mean frequency but progressively higher intraburst frequencies. $B$, Histogram of the average ratio of action potential integral during trains with different intraburst frequencies relative to that of the mean frequency train at the soma and distal dendritic sites. $C$, Spatial profile of voltage-dependent amplification of single action potentials. The relative amplitude of action potentials recorded at depolarized and resting membrane potentials is plotted as a function of somatodendritic recording location. Data were approximated with an arbitrary second order polynomial function constrained at the level of the soma. The inset shows that the amplitude of dendritic action potentials (600 $\mu \mathrm{m}$ from the soma) was dramatically increased during tonic depolarization $(-51 \mathrm{mV})$ from the resting membrane potential $(-62 \mathrm{mV})$. Calibration: $4 \mathrm{msec}$, $15 \mathrm{mV}$. correlation between distal dendritic action potential amplitude and instantaneous frequency was however not apparent, as groups of action potentials (two to five) generated at high frequency were required for the generation of action potential amplification (Fig. $2 C)$. Furthermore, dendritic action potential amplitude was depressed after periods of amplification (Fig. $2 C$ ), indicating that inactivation processes also contribute to this relationship (see below).

To examine the frequency dependence of distal dendritic action potential amplification in more detail we generated short trains of action potentials at low frequency $(10 \mathrm{~Hz})$ or as two action potential burst discharges with various intraburst frequencies that maintained the mean firing rate (Fig. $3 A$ ). As the intraburst frequency of trains was increased to $80 \mathrm{~Hz}$, the second dendritic action potential of each burst was found to be relatively amplified (Fig. $3 A$ ). These data were summarized by determining the ratio of the integral of action potential trains composed of burst discharges compared to mean trains. At $80 \mathrm{~Hz}$ the integral ratio of dendritically recorded responses was significantly $(p<0.05)$ greater than those generated at lower intraburst frequencies $(n=6$; Fig. $3 B)$. These data suggest that supralinear summation of dendritically recorded backpropagating action potentials underlies the relative amplification of physiological spike trains.

To explore the voltage-dependent nature of dendritic action potential amplification during physiological spike trains in more detail, we recorded single action potentials at various somatodendritic locations at resting and depolarized membrane potentials $(n=65$; Fig. $3 C)$. Tonic membrane depolarization was found to generate a dramatic amplification of dendritically recorded action potentials, which was accompanied by the appearance of a large secondary calcium component to the dendritic action potential (Kim and Connors, 1993; Stuart et al., 1997a; Larkum et al., 1999a,b; Williams and Stuart, 1999) (Fig. 3C, inset). As with the amplification of physiological spike trains, amplification of single action potentials was more pronounced at distal dendritic recording sites reaching values of fourfold to sevenfold at sites $>600 \mu \mathrm{m}$ from the soma. In fact, the spatial profile of voltage-dependent amplification of dendritic action potentials was strikingly similar to the spatial profile of amplification of physiological spike trains (compare Figs. $1 C, 3 C$ ). Together these data indicate that the supralinear temporal summation of action potentials evoked during physiological patterns of action potential firing is generated by a voltage-dependent amplification mechanism.

\section{Activity dependence of action potential backpropagation}

To gain further insights into the mechanisms shaping the activity dependence of action potential backpropagation during physiological patterns of action potential activity, we generated short trains of action potentials at different mean frequencies $(4.4-100 \mathrm{~Hz}, 10$ action potentials in each trial). At the level of the soma $(n=5)$ and in the proximal portion of the apical dendrite $(n=5 ;<300 \mu \mathrm{m}$ from the soma), action potentials showed little activity-dependent depression (Fig. 4A,B). In contrast, at more distal recording sites we observed a complex relationship between frequency- and activity-dependent modification of action potential backpropagation. At intermediate dendritic sites $(n=9 ; 300-480 \mu \mathrm{m}$ from the soma) action potentials evoked at low frequencies $(<10 \mathrm{~Hz})$ backpropagated reliably but showed progressively more activitydependent depression at higher frequencies. This depression was, however, relieved at the highest frequencies tested (Fig. 4A). This behavior is reflected in the summary data calculated by taking the cumulative action potential amplitude at each frequency for a number of neurons during recordings at intermediate dendritic locations (Fig. 4B). At the most distal dendritic recording sites $(n=$ 8 ; $>480 \mu \mathrm{m}$ from the soma) a different pattern of activitydependent modification emerged. Action potentials evoked at low frequencies were consistently of small amplitude throughout the train, however, when generated at high frequency the amplitude of distal dendritic action potentials first increased because of supralinear temporal summation, but then decreased, indicating a progressive failure of action potential backpropagation (Fig. 4A,B). Dendritic membrane depolarization at these distal sites led to enhanced backpropagation at both low and high frequencies $(n=$ 


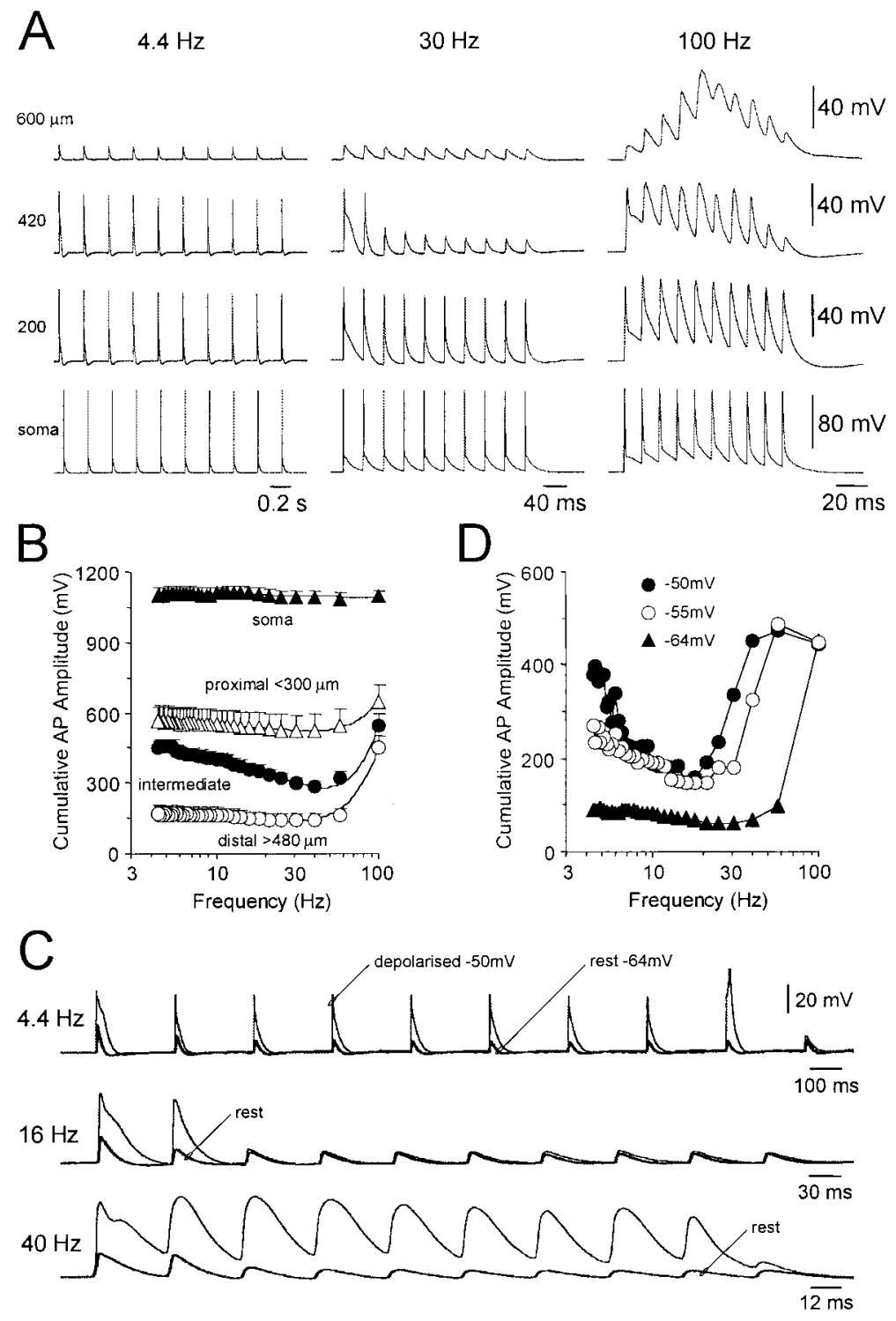

Figure 4. Frequency and voltage dependence of dendritic action potential backpropagation. $A$, Representative traces demonstrating the spatial pattern of action potential modification during regular spike trains presented at low, medium, and high frequencies $(4.4,30$, and $100 \mathrm{~Hz})$. Recordings were made at the indicated somatodendritic locations. Note at the most distal recording site the progressive amplification followed by depression of action potentials during a $100 \mathrm{~Hz}$ train and at more proximal recording locations the frequency-dependent depression at lower frequencies. $B$, Summary data demonstrating the frequency and spatia dependence of activity-dependent modification of action potential backpropagation. Data represent averaged values of cumulative action potential amplitude for each frequency of presentation $(10$ action potentials) recorded at somatic and different dendritic sites. The recording regions are indicated. Data were fit with linear regression analysis for somatic and with arbitrary second order polynomial functions for dendritic sites. $C$, Voltage dependence of activity-dependent modification. Representative distal dendritic recording (700 $\mu \mathrm{m}$ from the soma) demonstrating that dendritic membrane depolarization transforms the pattern of activitydependent depression, allowing faithful backpropagation of action potentials over a wider frequency range. Traces obtained at resting $(-64 \mathrm{mV}$; thick lines) and depolarized $(-50 \mathrm{mV}$; thin lines $)$ potentials have been overlain. $D$, Representative example of the voltagedependent transformation of the frequency dependence of activity-dependent action potential backpropagation at a distal dendritic recording location ( $700 \mu \mathrm{m}$ from the soma; same cell as in $C$ ). Relationships were constructed from the resting membrane potential $(-64 \mathrm{mV})$ and at the indicated depolarized membrane potentials.

$5 ;>500 \mu \mathrm{m}$ from the soma; Fig. $4 C, D)$. These data provide further evidence that action potential backpropagation is enhanced at high frequencies and that the frequency range over which action potentials faithfully backpropagate into the distal dendrites is voltage-dependent.

\section{Time course of recovery following activity-dependent depression of backpropagating action potentials}

Previous investigations have indicated that sustained highfrequency action potential firing leads to a progressive decrease in dendritic action potential amplitude in cortical neurons (Callaway and Ross, 1995; Spruston et al., 1995). At the level of the soma, action potential amplitude was maintained during sustained highfrequency activity ( 20 action potentials; $100 \mathrm{~Hz}$; Fig. $5 A$ ). At distal dendritic recordings sites ( $>400 \mu \mathrm{m}$ from the soma), however, the amplitude of action potentials was found to first increase because of the supralinear temporal summation of action potentials, but then sharply decrease during sustained activity, indicating a progressive failure of action potential amplification (Fig. 5A). These data indicate that the supralinear amplification of distal dendritic action potentials during high-frequency firing cannot be maintained over long periods of sustained activity. This effect will influence backpropagation during physiological action potential firing patterns and suggests that action potentials will fail to invade apical dendrites after sustained high-frequency firing.
We therefore mapped the time course of recovery from activitydependent depression by evoking single action potentials at times following sustained high-frequency activity. Consistent with the lack of activity-dependent depression at the level of the soma, action potential amplitude was found to be constant after sustained activity (Fig. 5A,B). At dendritic sites, however, recovery from activity-dependent depression was found to be progressively slower at more distal dendritic locations, with full recovery of action potential amplitude taking up to $1 \mathrm{sec}$ at dendritic sites 300-400 $\mu \mathrm{m}$ from the soma (Fig. 5A,B). At the most distal dendritic locations examined, recovery was slightly accelerated, presumably as recruitment (and subsequent inactivation) of distal dendritic sodium channels was reduced because of increased failure of action potential backpropagation during sustained high-frequency activity at these locations. Together these data indicate that at distal dendritic locations sustained high-frequency firing is followed by a period of relative refractoriness, which will impact on the pattern of dendritic activity evoked during physiological trains of action potential firing.

\section{Activity-dependent modification of sodium currents}

In CA1 pyramidal neurons activity-dependent attenuation of backpropagating action potentials is generated by the progressive inactivation of dendritic sodium channels (Spruston et al., 1995; Colbert et al., 1997; Jung et al., 1997). To test whether this is also the 

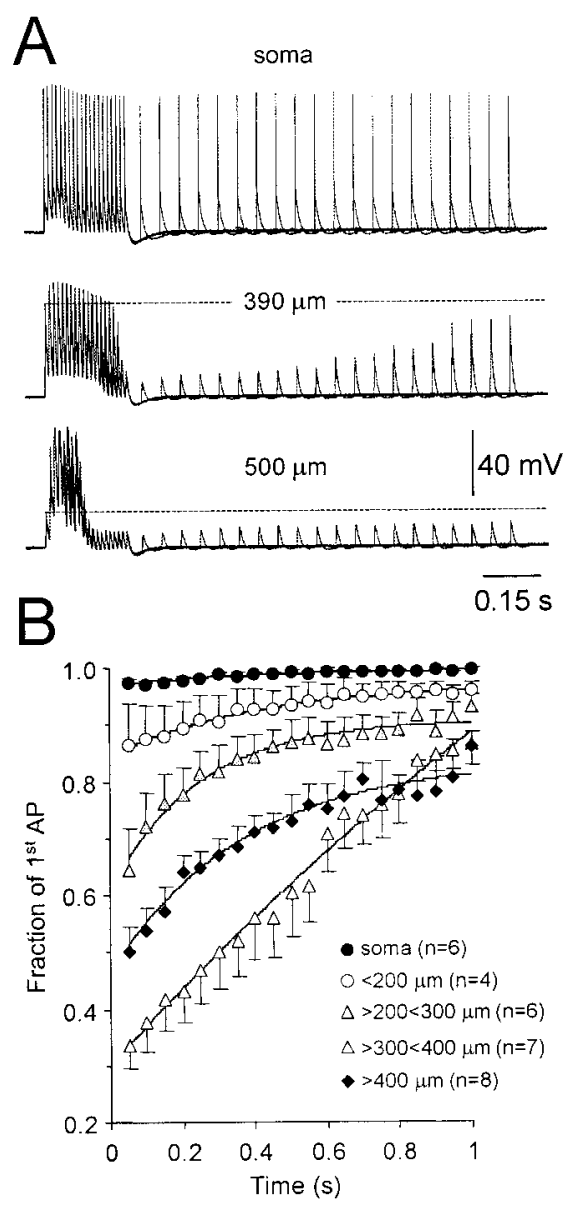

Figure 5. Recovery from activity-dependent depression. $A$, Representative traces showing the spatial pattern of action potential recovery following sustained high-frequency firing ( 20 action potentials at $100 \mathrm{~Hz}$ in each trial, 21 overlain traces). Recordings were made at the indicated somatodendritic locations. Note the failure of action potential backpropagation during sustained firing and the slow recovery of action potential amplitude at distal dendritic sites. $B$, Summary data showing the spatial profile of the rates of recovery of action potential amplitude after sustained action potential firing. In each neuron, action potential amplitudes have been normalized to the amplitude of the first action potential of the conditioning highfrequency train. Recordings made at similar somatodendritic locations were pooled. Data have been fit with single exponentials (solid lines).

case in layer 5 neocortical pyramidal neurons, we examined the activity-dependent inactivation of ensemble sodium channel currents from axonal, somatic, and apical dendritic locations using cell-attached recording techniques. We tested for progressive sodium channel inactivation by generating sodium channel activity in response to a train of $10,2 \mathrm{msec}, 60 \mathrm{mV}$ voltage steps from the resting membrane potential at frequencies of $10-100 \mathrm{~Hz}$ (Fig. 6A). Even at the highest frequency tested $(100 \mathrm{~Hz})$ we observed only a modest progressive decrease in channel activity during the test train at all recording locations (Fig. 6A,B). The degree of activitydependent inactivation was quantified by comparing the amplitude of the tenth and first current responses of each trial. Across the entire axodendritic area examined we observed a roughly constant level of inactivation during $100 \mathrm{~Hz}$ trains of $23.9 \pm 0.9 \%(n=50$; Fig. $6 C$ ), whereas, as reported previously, the amplitude of sodium channel activity was reasonably constant across the axodendritic area examined (Fig. 6D) (Stuart and Sakmann, 1994). The degree of activity-dependent inactivation of sodium channel activity was significantly less $(p<0.05)$ at lower frequencies, reaching values of $14.7 \pm 2.1 \%$ at $10 \mathrm{~Hz}$ (Fig. $6 F$ ). Importantly, we observed that the degree of dendritic sodium channel inactivation was lowest when we replicated action potential burst experiments, like those shown in Figure $3 A$ (average rate, $10 \mathrm{~Hz}$; intraburst frequency, $80 \mathrm{~Hz}$; Fig.

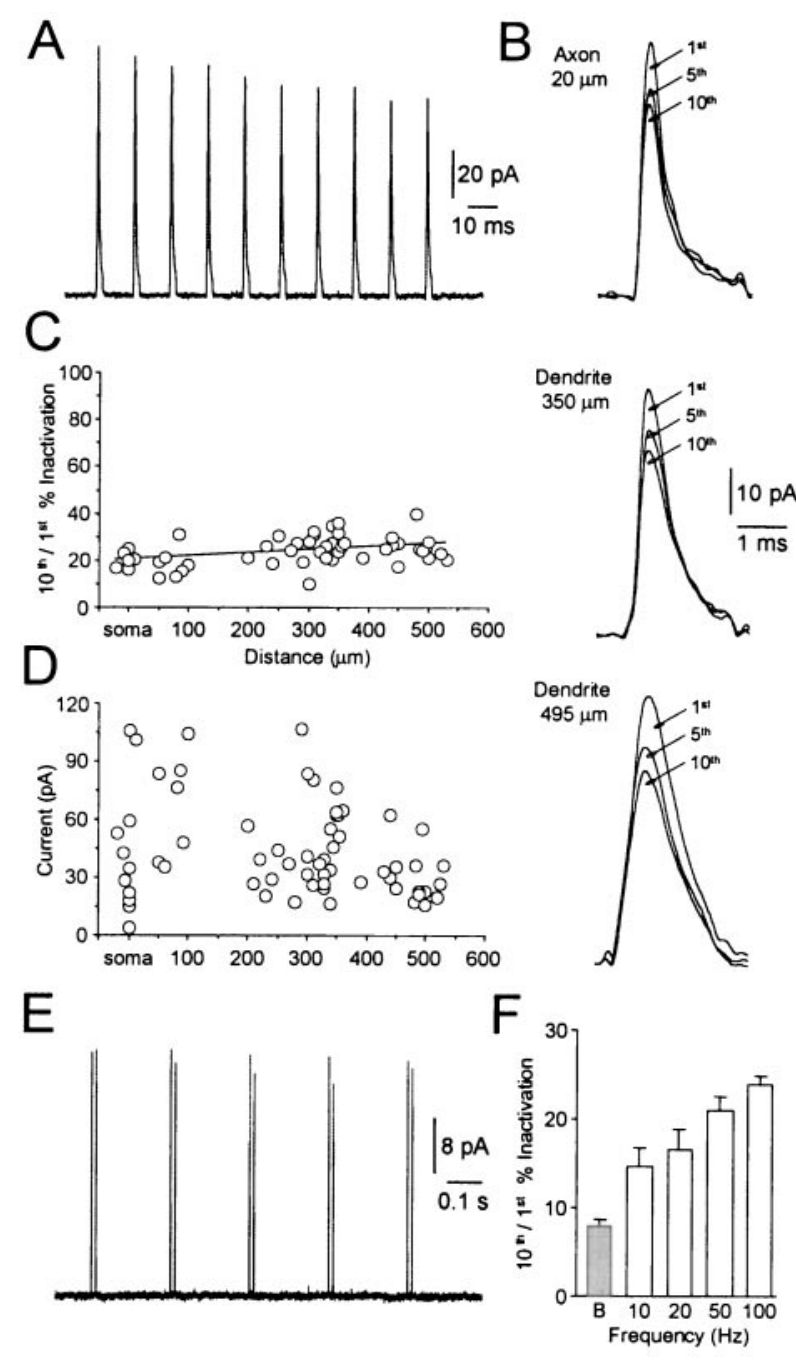

Figure 6. Frequency dependence of ensemble sodium currents. A, Cellattached recording of ensemble sodium channel activity generated by 10,2 msec, $60 \mathrm{mV}$ voltage steps repeated at a frequency of $100 \mathrm{~Hz}$. Average of 20 trials at the soma. Note the small progressive decrease in current amplitude throughout the train. $B$, Similar levels of progressive sodium channel inactivation were observed at axonal and two dendritic recording sites. The first, fifth, and tenth current response to 10 voltage steps at $100 \mathrm{~Hz}$ are shown at a fast time base. Average of 20 trials. $C$, Summary data indicating a similar level of progressive sodium channel inactivation across the axodendritic area examined. Values represent the percentage reduction of the tenth response compared to the first during $100 \mathrm{~Hz}$ trains at different distances from the soma. The data were fit with a linear regression. $D$, Pooled data illustrating the axodendritic distribution of ensemble sodium channel activity during $60 \mathrm{mV}, 10 \mathrm{msec}$ steps from the resting membrane potential. Note the high degree of variability from patch to patch, but the lack of obvious spatial dependence. $E$, Cell-attached recording demonstrating minimal sodium channel inactivation during bursts. Currents were evoked at a mean frequency of $10 \mathrm{~Hz}$ with an intraburst frequency of $80 \mathrm{~Hz}$. Dendritic recording $485 \mu \mathrm{m}$ from the soma. $F$. Frequency dependence of sodium channel inactivation. Average percentage reduction of the tenth sodium current compared to the first at the indicated frequencies. The gray bar indicates the percentage reduction of the second action potential of each burst compared to the first during a burst paradigm as in $E$.

$6 E, F)$. Under these conditions, we observed that the second current response during a burst was attenuated relative to the first current response of each burst by on average only $7.9 \pm 0.8 \%(n=$ 6; average recording distance from soma, $360 \mu \mathrm{m}$; Fig. $6 F$ ).

\section{Amplification of physiological spike trains is generated by the recruitment of dendritic voltage-activated channels}

To investigate the role of dendritic sodium channels in the amplification of physiological patterns of action potential firing, we 
Figure 7. Blockade of distal dendritic sodium channels attenuates amplification of physiological spike trains. $A$, Dendritic action potentials ( $620 \mu \mathrm{m}$ from the soma) evoked during physiological (top traces) and mean spike trains (middle traces) under control conditions (thin lines) and during local dendritic application of TTX (1 $\mu \mathrm{m})$ (thick lines). Note that local TTX application greatly attenuates dendritic action potentials during physiological but not mean spike trains. The bottom trace shows the dendritic action potentials evoked during physiological spike trains recorded at a hyperpolarized membrane potential. The physiological spike train had a mean frequency of $50 \mathrm{~Hz}$ and $\mathrm{CV}$ of 1.01. The experimental arrangement is summarized in the inset. $B$, Summary data demonstrating that local TTX application greatly reduced the integral of dendritic spike trains. Data expressed as a fraction of control. $C$, Local dendritic TTX application blocks the voltage-dependent amplification of single dendritic action potentials. Dendritic action potentials were evoked from resting $(-62 \mathrm{mV})$ and from depolarized $(-48$ $\mathrm{mV}$ ) membrane potentials under control conditions (thin lines) and in the presence of local TTX (thick lines). Dendritic recording 590 $\mu \mathrm{m}$ from soma. $D$, Summary data indicating that local dendritic TTX application failed to alter the amplitude of single dendritic action potentials recorded at the resting membrane potential, but greatly reduced the amplitude of action potentials evoked from depolarized potentials (shaded bar). Values are expressed as a fraction of control. $E$, Dendritic action potentials $(480 \mu \mathrm{m}$ from the soma) evoked during a physiological spike train (mean frequency of $73 \mathrm{~Hz}$ and $\mathrm{CV}$ of 1.47) under control conditions (thin line) and during bath application of cadmium $(100 \mu \mathrm{m})$ (thick line). action potentials during physiological (shaded bar) but not mean
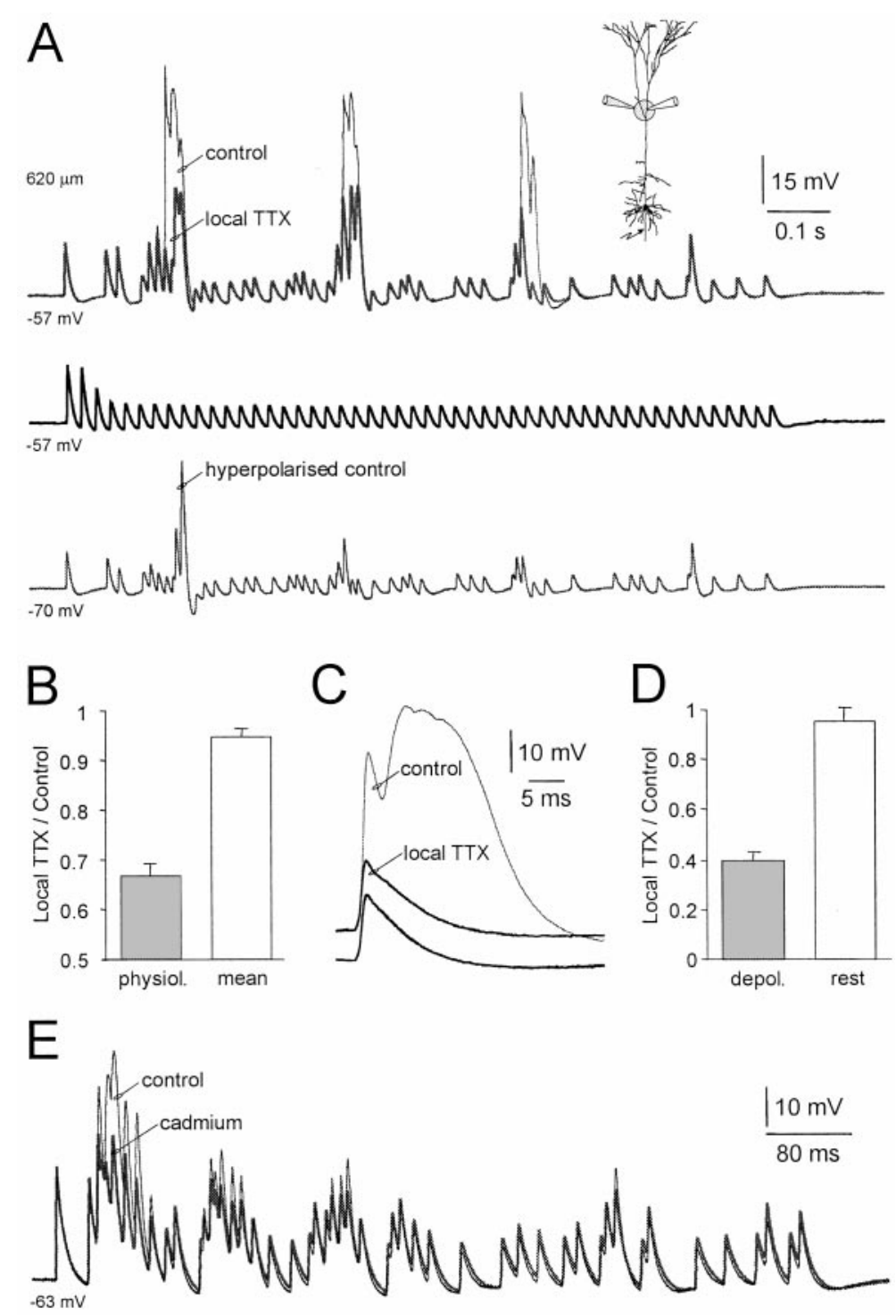

locally applied the sodium channel blocker TTX close to the dendritic recording location $(n=10$; average recording distance from soma $542 \mu \mathrm{m}$; Fig. 7A). Local application of TTX substantially reduced the amplitude of dendritic electrogenesis during physiological spike trains, but did not alter the amplitude of dendritic action potentials during mean trains (Fig. 7A). On average the local application of TTX significantly $(p<0.05)$ reduced the integral of physiological spike trains by $36 \pm 2 \%$, while not significantly effecting the integral of mean trains $(5 \pm 2 \%$ reduction; $n=$ 10; Fig. 7B). By comparison, in a group of these neurons dendritic membrane potential hyperpolarization led to a reduction in the integral of physiological spike trains by $63 \pm 3 \%(n=4$; Fig. $7 A)$. We also explored if local application of TTX could block the voltage-dependent amplification of single dendritic action potentials (Fig. $7 C$ ). At distal dendritic sites $(n=6$; average recording distance from soma $550 \mu \mathrm{m}$ ) local application of TTX greatly reduced the amplitude of dendritic action potentials evoked from depolarized potentials by $60 \pm 3 \%$, but failed to alter the amplitude of dendritic action potentials recorded at resting potentials $(4 \pm$ $5 \%$ reduction; Fig. $7 C, D$ ). These data directly demonstrate that the amplification of physiological spike trains involves the recruitment of distal dendritic sodium channels.

The greater reduction of physiological spike trains achieved by direct dendritic membrane potential hyperpolarization compared to local blockade of distal dendritic sodium channels suggests that other voltage-activated currents maybe involved in their amplifica- tion. To test for the involvement of calcium channels we bath applied the nonspecific calcium channel antagonist cadmium (100 $\mu \mathrm{m})$. Application of cadmium led to a $25 \pm 10 \%$ decrease in the maximal amplitude of action potentials evoked during highfrequency components of physiological spike trains, but increased the integral of physiological spike trains by $19 \pm 8 \%(n=5$; average recording distance from soma $448 \mu \mathrm{m}$; Fig. $7 E$ ). The increase in the integral of spike trains, despite the reduction in peak amplitude, was generated as a consequence of an increase in the duration of low-amplitude action potentials. In accordance with this, the application of cadmium also increased the integral of spike trains reflecting the mean firing rate of physiological firing patterns by $54 \pm 3 \%(n=5$; average recording distance from soma $448 \mu \mathrm{m})$. These data indicate that cadmium has complex actions, presumably generated as a consequence of the blockade of dendritic calcium channels engaged during high-frequency action potential firing (Larkum et al., 1999a), and somatodendritic calcium channels that are linked to the generation of the action potential afterhyperpolarization (Schwindt et al., 1988; Williams and Stuart, 1999).

\section{DISCUSSION}

Action potentials actively propagate back into the dendritic tree of many neuronal types (Stuart et al., 1997b). In pyramidal neurons backpropagation is decremental and can lead to frequencydependent failure during action potential trains (Callaway and Ross, 1995; Spruston et al., 1995). This finding questions the 
importance of dendritic action potential backpropagation in information processing in the awake animal in which neurons often fire at high rates for prolonged periods. In contrast to previous studies describing activity-dependent depression of backpropagation during regular spike trains (Callaway and Ross, 1995; Spruston et al., 1995; Colbert et al., 1997; Jung et al., 1997), here we show that during high-frequency components of physiological spike trains action potentials backpropagate faithf ully into the distal dendrites. This occurs because of their supralinear temporal summation following recruitment of distal dendritic sodium and calcium channels. These findings indicate that variations in instantaneous frequency during physiological patterns of action potential firing have an important role in dendritic signaling.

\section{Backpropagation during action potential trains}

Our results suggest that the spatial decrement and failure of action potential backpropagation during trains is generated by the observed modest levels of progressive inactivation of dendritic sodium channels. Coupled with effects of dendritic morphology (Spruston et al., 1995; Hausser et al., 1998) at distal dendritic sites this leads to the failure of action potentials to attain the threshold level of depolarization necessary to recruit dendritic sodium and calcium channels. Active backpropagation can be restored, however, when dendritic action potentials summate temporally during physiological patterns of activity, high-frequency trains, or during bursts. Similarly, active backpropagation could be restored by dendritic depolarization. In all these situations restoration of active backpropagation occurs as passively spreading dendritic action potentials attained the threshold level of dendritic depolarization required for the regenerative activation of dendritic voltageactivated channels. The key role of dendritic voltage-activated channels in this mechanism was directly verified by the reduction of action potential amplification during physiological patterns of activity by dendritic membrane hyperpolarization (Fig. 2), the local blockade of dendritic sodium channels (Fig. 7), and by the blockade of calcium channels. We suggest that during high-frequency components of physiological firing patterns action potential amplification is initially generated by the recruitment of dendritic sodium channels. Amplified action potentials then in turn lead to the recruitment of dendritic calcium channels. This mechanism parsimoniously explains the amplification of action potentials at distal dendritic locations during physiological spike trains and indicates that in vivo backpropagation into the distal dendrites is likely to be more robust than previously thought.

Recent studies have demonstrated that brief high-frequency trains or bursts of action potentials in layer 5 pyramidal neurons are effective stimuli for the activation of dendritic calcium channels (Kim and Connors, 1993; Stuart et al., 1997a; Larkum et al., 1999a,b; Williams and Stuart, 1999). As physiological patterns of action potential firing contain high-frequency components, they would be expected to also activate dendritic calcium channels. Consistent with this we observed that the blockade of calcium channels decreased the supralinear temporal summation of dendritic action potentials during physiological spike trains. Based on previous observations, we suggest that calcium electrogenesis triggered by physiological patterns of action potential firing will be enhanced at distal apical dendritic locations (Schiller et al., 1997; Larkum et al., 1999a). Because recent observations have indicated that brief bursts of high-frequency firing induces large increases in intracellular calcium throughout the apical dendritic tree of layer 5 pyramidal neurons (Larkum et al., 1999a), a coherent increase in intracellular calcium would be expected to occur during highfrequency components of physiological firing patterns. Evidence that this is the case has recently been obtained during in vivo calcium-imaging experiments (Helmchen et al., 1999). As many forms of synaptic plasticity are dependent upon rises in intracellular calcium (Bliss and Collingridge, 1993), it is compelling to speculate that the high-frequency components of physiological spike trains will produce rises in dendritic calcium that may be involved in the long-term modification of synaptic strength.

\section{Factors controlling activity-dependent modification of action potential backpropagation}

We have revealed two factors that control the activity-dependent modification of action potential backpropagation during spike trains: (1) the recruitment of distal dendritic sodium and calcium channels when summation of backpropagating action potential reaches the threshold for their activation, and (2) the cumulative inactivation of dendritic sodium channels. These mechanisms will act in concert during trains of action potentials at distal dendritic locations, as frequency-dependent supralinear summation produced by the activation of distal dendritic sodium and calcium channels is checked by the cumulative inactivation of sodium channels, eventually leading to failure of active backpropagation. The interplay between these processes was directly demonstrated during long trains of high-frequency action potential firing (Fig. 5). The cumulative inactivation of sodium channels (Fig. 6) will act to control the ability of backpropagating action potentials to follow high-frequency components of physiological spike trains, such that at distal dendritic sites periods of intense dendritic depolarization during physiological spike trains will be followed by periods of relative refractoriness, the time course of which will be governed by the recovery of sodium channels from inactivation. Together these data indicate that effective backpropagation of action potentials throughout the apical dendritic tree of layer 5 neurons will only occur when neurons fire at high frequencies for relatively brief periods.

\section{Temporal versus rate coding}

The random nature of stimulus-evoked action potential trains in neocortical neurons in vivo does not result from noise within the spike generation mechanism (Mainen and Sejnowski, 1995; Holt et al., 1996; Nowak et al., 1997; Stevens and Zador, 1998). Variability must, therefore, arise from the temporal pattern of synaptic inputs and the postsynaptic mechanisms used to integrate them. Neocortical neurons have been suggested to employ one of two methods of synaptic integration, described as (1) coincidence detection, and (2) random walk, that in simple and complex neuronal models are capable of reproducing the irregular firing patterns observed in vivo (for review, see Shadlen and Newsome, 1994; Softky, 1995; Konig et al., 1996). Coincidence detection integrates temporally correlated synaptic inputs over short time periods $(<2 \mathrm{msec})$ to produce irregular action potential trains, where the timing of each action potential precisely reflects the temporal synchrony of specific synaptic inputs (Softky and Koch, 1993; Stevens and Zador, 1998; Harsch and Robinson, 2000). Random walk integration, on the other hand, describes integration of excitatory and inhibitory synaptic inputs over time scales defined by the membrane time constant, where the timing of each action potential is determined stochastically and information is represented as noisy average firing rates that are typically measured over hundreds of milliseconds (Shadlen and Newsome, 1994).

We have bypassed the integration of synaptic inputs in the present investigation to explore if the precise timing of action potentials has a specific dendritic signaling role. The observed reliance of effective dendritic action potential backpropagation on the timing and pattern of action potential initiation during physiological spike trains suggests that spike timing contains important signaling information. Previous work has indicated that the irregularity of in vivo firing patterns may be reproduced in neocortical neurons in vitro, if, and only if, neurons are driven with simulated excitatory and inhibitory synaptic inputs that contain a significant synchrony in their pattern of occurrence (Stevens and Zador, 1998; Harsch and Robinson, 2000), as suggested from modeling studies (Shadlen and Newsome, 1994; Softky, 1995). A consequence of input synchrony is to produce periods of high-frequency action potential firing that lend the spike train its variable frequency content and so apparent randomness (Stevens and Zador, 1998). Our results indicate that this synchronous synaptic input is transduced into powerful dendritic electrogenesis in neocortical layer 5 pyramidal neurons, implying a causal relationship between input 
synchrony and the generation of distal dendritic electrogenesis. On the other hand, if it is assumed that information is represented by mean firing rates measured over hundreds of milliseconds, the noise within this signal will lead to the generation of random dendritic electrogenesis. We suggest that if neurons signal by rate codes, action potential rates calculated over short time windows contain the important signaling information, as we observed that dendritic electrogenesis was most effectively engaged during periods of high-frequency action potential firing. Consistent with this idea, bursts of action potentials have been shown to posses significant informational content (Livingstone et al., 1996; Lisman, 1997), and in vivo rate codes calculated over short periods $(<50$ $\mathrm{msec}$ ) have been found to signal stimulus characteristics almost as efficiently as rate codes calculated over hundreds of milliseconds (Tovee et al., 1993).

In conclusion, we find that periods of high-frequency firing within physiological action potential trains give rise to amplification of backpropagating action potentials in distal dendrites. The apparently random firing pattern of cortical neurons may therefore contain information that posses a dendritic signaling role and so should not be considered as noise around a mean action potential firing rate. Rather, in neocortical layer 5 pyramidal neurons, the high-frequency components of physiological spike trains, generated by synchronous activation of synaptic inputs, lead to a powerful retrograde signal that is transmitted throughout the dendritic tree.

\section{REFERENCES}

Bliss TV, Collingridge GL (1993) A synaptic model of memory: long-term potentiation in the hippocampus. Nature 361:31-39.

Buzsaki G, Kandel A (1998) Somadendritic backpropagation of action potentials in cortical pyramidal cells of the awake rat. J Neurophysiol 79:1587-1591.

Callaway JC, Ross WN (1995) Frequency-dependent propagation of sodium action potentials in dendrites of hippocampal CA1 pyramidal neurons. J Neurophysiol 74:1395-1403.

Colbert CM, Magee JC, Hoffman DA, Johnston D (1997) Slow recovery from inactivation of $\mathrm{Na}+$ channels underlies the activity-dependent attenuation of dendritic action potentials in hippocampal CA1 pyramidal neurons. J Neurosci 17:6512-6521.

Fleidervish IA, Friedman A, Gutnick MJ (1996) Slow inactivation of Na+ current and slow cumulative spike adaptation in mouse and guinea-pig neocortical neurones in slices. J Physiol (Lond) 493:83-97.

Harsch A, Robinson HP (2000) Postsynaptic variability of firing in rat cortical neurons: the roles of input synchronization and synaptic NMDA receptor conductance. J Neurosci 20:6181-6192.

Hausser M, Vetter P, Roth A (1998) Action potential backpropagation depends on dendritic geometry. Soc Neurosci Abstr 24:1813.

Helmchen F, Svoboda K, Denk W, Tank DW (1999) In vivo dendritic calcium dynamics in deep-layer cortical pyramidal neurons. Nat Neurosci 2:989-996.

Holt GR, Softky WR, Koch C, Douglas RJ (1996) Comparison of discharge variability in vitro and in vivo in cat visual cortex neurons. J Neurophysiol 75:1806-1814.

Johnston D, Magee JC, Colbert CM, Cristie BR (1996) Active properties of neuronal dendrites. Annu Rev Neurosci 19:165-186.

Jung HY, Mickus T, Spruston N (1997) Prolonged sodium channel inactivation contributes to dendritic action potential attenuation in hippocampal pyramidal neurons. J Neurosci 17:6639-6646.

Kim HG, Connors BW (1993) Apical dendrites of the neocortex: correlation between sodium- and calcium-dependent spiking and pyramidal cell morphology. J Neurosci 13:5301-5311.
Konig P, Engel AK, Singer W (1996) Integrator or coincidence detector? The role of the cortical neuron revisited. Trends Neurosci 19:130-137.

Larkum ME, Kaiser KM, Sakmann B (1999a) Calcium electrogenesis in distal apical dendrites of layer 5 pyramidal cells at a critical frequency of back-propagating action potentials. Proc Natl Acad Sci USA 96:14600-14604.

Larkum ME, Zhu JJ, Sakmann B (1999b) A new cellular mechanism for coupling inputs arriving at different cortical layers. Nature 398:338-341.

Lisman JE (1997) Bursts as a unit of neural information: making unreliable synapses reliable. Trends Neurosci 20:38-43.

Livingstone MS, Freeman DC, Hubel DH (1996) Visual responses in V1 of freely viewing monkeys. Cold Spring Harb Symp Quant Biol 61:27-37.

Magee JC, Johnston D (1997) A synaptically controlled, associative signal for Hebbian plasticity in hippocampal neurons. Science 275:209-213.

Mainen ZF, Sejnowski TJ (1995) Reliability of spike timing in neocortical neurons. Science 268:1503-1506.

Nowak LG, Sanchez-Vives MV, McCormick DA (1997) Influence of low and high frequency inputs on spike timing in visual cortical neurons. Cereb Cortex 7:487-501.

Pare D, Shink E, Gaudreau H, Destexhe A, Lang EJ (1998) Impact of spontaneous synaptic activity on the resting properties of cat neocortical pyramidal neurons In vivo. J Neurophysiol 79:1450-1460.

Schiller J, Schiller Y, Stuart G, Sakmann B (1997) Calcium action potentials restricted to distal apical dendrites of rat neocortical pyramidal neurons. J Physiol (Lond) 505:605-616.

Schwindt PC, Spain WJ, Foehring RC, Stafstrom CE, Chubb MC, Crill WE (1988) Multiple potassium conductances and their functions in neurons from cat sensorimotor cortex in vitro. J Neurophysiol 59:424-449.

Shadlen MN, Newsome WT (1994) Noise, neural codes and cortical organization. Curr Opin Neurobiol 4:569-579.

Shadlen MN, Newsome WT (1995) Is there a signal in the noise? Curr Opin Neurobiol 5:248-250.

Shadlen MN, Newsome WT (1998) The variable discharge of cortical neurons: implications for connectivity, computation, and information coding. J Neurosci 18:3870-3896.

Softky WR (1995) Simple codes versus efficient codes. Curr Opin Neurobiol 5:239-247.

Softky WR, Koch C (1993) The highly irregular firing of cortical cells is inconsistent with temporal integration of random EPSPs. J Neurosci $13: 334-350$.

Spruston N, Schiller Y, Stuart G, Sakmann B (1995) Activity-dependent action potential invasion and calcium influx into hippocampal CA1 dendrites. Science 268:297-300.

Stevens CF, Zador AM (1998) Input synchrony and the irregular firing of cortical neurons. Nat Neurosci 1:210-217.

Stuart GJ, Sakmann B (1994) Active propagation of somatic action potentials into neocortical pyramidal cell dendrites. Nature 367:69-72.

Stuart G, Sakmann B (1995) Amplification of EPSPs by axosomatic sodium channels in neocortical pyramidal neurons. Neuron 15:1065-1076.

Stuart G, Schiller J, Sakmann B (1997a) Action potential initiation and propagation in rat neocortical pyramidal neurons. J Physiol (Lond) 505:617-632.

Stuart G, Spruston N, Sakmann B, Häusser M (1997b) Action potential initiation and backpropagation in neurons of the mammalian CNS. Trends Neurosci 20:125-131.

Tovee MJ, Rolls ET, Treves A, Bellis RP (1993) Information encoding and the responses of single neurons in the primate temporal visual cortex. J Neurophysiol 70:640-654.

Williams SR, Stuart GJ (1999) Mechanisms and consequences of action potential burst firing in rat neocortical pyramidal neurons. J Physiol (Lond) 521:467-482.

Williams SR, Stuart GJ (2000) Action potential backpropagation and somato-dendritic distribution of ion channels in thalamocortical neurons. J Neurosci 20:1307-1317. 